\title{
Una rete castellare: il sistema fortificato irpino
}

\author{
A network of castles: the Irpinian fortified system
}

\section{Giovanni Coppola}

Università di Napoli “Suor Orsola Benincasa”, Naples, Italy, giovanni.coppola@unisob.na.it

\begin{abstract}
In Irpinia, to grasp the extent to which the multiform and varied castellated density still has today, it is necessary to look at its hilly and mountainous landscapes or read the toponyms of its villages: from the recent study carried out in the Province of Avellino, there is a list of 78 castles still visible in elevation, a very high figure if you consider that the entire provincial territory is composed of 118 municipalities, for a percentage of almost $70 \%$.

The period of study in which this research is based on Irpinia's fortification (castles, walls, towers and defensive walls) is part of the period in which the various foreign dynasties conquered the Regnum Siciliae giving rise to a military architecture which goes from the Longobard domination (568-774) to the advent of the Normans (1130-1194) and the Swabians (1194-1268), to retrace the phases of the coming first of the Angevins (1268-1435) and then of the Aragons (1442-1503).
\end{abstract}

Keywords: castle, fortification, military architecture, medieval architecture, South Italy, Irpinia.

\section{L'armatura del sistema difensivo medievale irpino}

L'Irpinia è una multiforme e variegata "terra di castelli" che ha risentito enormemente dei destini dei tanti conquistatori e delle visioni politiche che ne hanno disegnato i confini e guidato le sorti.

Dal censimento effettuato, che riguarda un arco temporale di circa dieci secoli, risulta un elenco di 78 castelli, una cifra altissima se si pensa che la Provincia di Avellino è composta da 118 Comuni, per una percentuale di quasi il $70 \%$ dell'intero territorio.

\section{Torri, castelli e fortificazioni longobarde}

Il grande patrimonio culturale irpino risiede soprattutto nella ricchezza, su tutto il territorio, delle testimonianze materiali medievali, non solo architettoniche ma anche archeologiche e storico-artistiche (Gandolfo, Muollo, 2013; Cucci- niello, 2012). Durante gli anni successivi alla caduta dell'Impero romano, il cosiddetto fenomeno dell'incastellamento, dopo che lentamente i centri antichi di fondovalle si spopolavano, iniziò a modificare profondamente l'originaria conformazione morfologica del territorio con la nascita di siti difensivi sulle alture e sui rilievi rocciosi, ovvero su spazi protetti naturalmente e da cui era possibile controllare fisicamente le valli, le pianure circostanti e le strade di comunicazione ${ }^{1}$.

Tutto ciò fu reso possibile dalla cultura di un popolo che, con diverse ed alterne vicende, per ben cinque secoli qui ebbe dimora e governo: i Longobardi (Brogiolo, Marazzi, Giostra, 2017; VV.AA., 2003).

Una delle testimonianze architettoniche più rilevanti di questo periodo è la costruzione, databile 
tra VII e IX secolo, come attestano le tipologie murarie e gli stessi ritrovamenti ceramici del tipo a bande rosse, del recinto fortificato della Civita di Ogliara (Peduto, 2007, pp. 415, 430-431, 434), nel territorio di Serino, a poca distanza dalle sorgenti del fiume Sabato. Il sito sorge lungo la fascia pedemontana del Monte Terminio e si presenta come una fortificazione costituita da mura lunghe circa due chilometri, con una forma irregolare che segue l'andamento del promontorio.

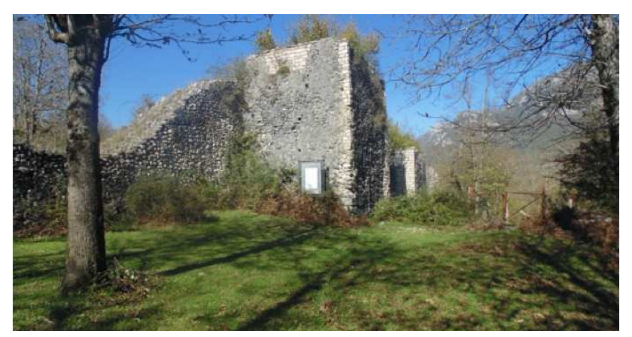

Fig. 1. Serino, Civita di Ogliara, recinto fortificato, epoca longobarda.

Un altro nodo viario importante era costituito dal passo della Sella di Conza, in Alta Irpinia, che domina le valli dei fiumi Ofanto e Sele, da dove era possibile giungere facilmente a Benevento e a Capua (Johannowsky, 2000). Nel 591, i Longobardi guidati da Arechi I, costrinsero i Bizantini alla resa e consolidarono il centro fortificato di Conza, come presidio posto a difesa della capitale del ducato. Da qui partì la costruzione di una cortina difensiva composta dai castelli di Calitri, Cairano, Morra de Sanctis, Teora e Viarum nel territorio di Caposele (Coppola, Muollo, 2017, pp. 162-163, 155-157, 264-266, 169-170), una località oggi conosciuta con il nome di Pietra Boiara, sulla cui roccia sorgeva un fortilizio del quale avanzano pochi ruderi e che un tempo funzionava proprio da avamposto di controllo e di avvistamento per l'importante valico di Conza.

Anche la fortificazione della rupe di Quaglietta (Coppola, Muollo, 2017, pp. 162-163; Putaturo Donati Viscido di Nocera, 2008, pp. 19-25, 44), baricentro dell' alta valle del Sele, deve essere ricondotta alla prima fase dell'occupazione longobarda, nonostante le superfetazioni di età normanna e sveva impediscano la lettura precisa della prima fase dell'insediamento.
La Radelgisi et Siginulfi principum divisio ducatus Beneventani dell'849 (Martin, 2005, pp. 201217), voluta dall'imperatore Lotario II, al termine del conflitto decennale tra Benevento e Salerno (839-849), portò ad accrescere la rete difensiva castellare. Con questo nuovo assetto politico, infatti, si infrangeva la secolare unità dello stato beneventano e si assisteva, di fatto ad una vera e propria guerra fratricida, che portò ad una nuova e più frazionata spartizione di terre da parte di un' aristocrazia potenziata di numero e divisa in due gruppi, fautori dei loro rispettivi capi (Erchemperto, 1999, p. 52). Alla fine delle varie vicende storiche il risultato fu la nascita di due principati: quello di Salerno, assegnato a Siconolfo, con sbocchi sul mare e sulle pianure, e quello di Benevento, assegnato a Radelchi, limitato alle regioni interne del Sannio, dell'Irpinia e del Molise. L'Irpinia, inglobata nel ducato Beneventano, fu divisa in gastaldati, ossia distretti amministrativi, cui fu preposto un funzionario, il gastaldo, al quale competevano funzioni amministrative, poteri giurisdizionali e di organizzazione militare. La regione appare così, tra il IX e il XIII secolo, costellata da una miriade di piccoli centri fortificati, alcuni a difesa dei gastaldati di Montella e di Conza, entrati a far parte del principato di Salerno, altri invece a guardia dei gastaldati di Quintodecimo (l'odierna Mirabella Eclano) e di Ariano, appartenenti al principato di Benevento, e poi impianti fortificati sorti a protezione delle zone interne poste lungo il crinale tra la valle del Sabato e del Calore e, infine, fortilizi costruiti a difesa del gastaldato di Nola, come Lauro, Forino, Serino e Avella (Cinquantaquattro, Camardo, Basile, 2003, pp. 355-361).

Anche se alcuni tratti delle frontiere tracciate dalla divisio tra i principati di Benevento, Salerno appaiono chiari in altri, invece, risulta molto difficile risalire ai confini territoriali precisi dei diversi gastaldati (Gasparri, 1995, pp. 9-19; Martin, 1992, pp. 259-276).

Il principe di Salerno provvide immediatamente a fortificare la zona montuosa alle spalle della città, dalle serre di Montoro, ai monti Terminio e Cervialto. Alcuni insediamenti furono fondati $e x$ novo con lo scopo di controllare le vie di accesso alla Puglia e alla piana del Sele: è questo il caso di tre insediamenti della provincia di Avellino, 
Torella dei Lombardi, Guardia dei Lombardi e Sant'Angelo dei Lombardi (Coppola, Muollo, 2017, pp. 207-209; Rotili, 2002, pp. 205-240).

Il principe di Benevento, fece fortificare il territorio di Avellino (tra le valli del Sabato e del Calore) e tutto il confine orientale, occupato dal gastaldato di Quintodecimo, con i castelli di Frigento, Villamaina, Paternopoli, San Mango sul Calore e Rocca San Felice (Coppola, Muollo, 2017, pp. 196-197, 274-275, 295-299; Rotili, 1991-1992a, pp. 231-384), posti nelle zone frontaliere. Anche il fortilizio di Sant'Angelo al Pesco (Coppola, Muollo, 2017, pp. 196-197; Gambino, 1977, pp. 13-19), nell'attuale territorio di Frigento, e il castello di Monticchio (o Monticchio dei Lombardi) (Colantuono, 1993, pp. 1322), che sorgeva su un'altura immediatamente a nord dell'abbazia del Goleto, furono edificati nella seconda metà del IX secolo lungo il confine tra il ducato longobardo di Benevento e quello di Salerno.

Ad ogni modo possiamo affermare che dopo la conquista longobarda del VI secolo, alcuni insediamenti scomparvero del tutto o conobbero un lungo periodo di declino, altri si potenziarono oppure videro la luce proprio tra i secoli VI-IX (Coppola, Muollo, 2017, pp. 133-136, 234-236, 310-312). In effetti l'incastellamento longobardo si attuò per mezzo di due momenti: da un lato, lo spostamento di uomini in un unico abitato in altura e appositamente difeso, attorno a cui gravitava un territorio compatto in cui si procedette allo sviluppo delle colture; dall'altro, per esigenze squisitamente militari, si procedette all'erezione di castelli, in primo luogo per fissare l'avvenuto dominio di uno specifico territorio e, successivamente, per rispondere a esigenze di demarcazione dei confini delle diverse aree di influenza signorili, in caso di invasioni nemiche, e per controllare importanti vie di transito (Toubert, 1997).

\section{Torri, castelli e fortificazioni normanne}

L'opera di incastellamento fu consolidata e incrementata durante la dominazione normanna (Coppola, 2015, pp. 33-48; Coppola, 2005, pp. 1-50) e, come si evince dal Catalogus Baronum (Jamison, 1972; Cuozzo, 1985), l'originario scacchiere castellare longobardo restò pressoché invariato: le nuove contee ricalcarono spesso la precedente suddivisione dotandosi di un nuovo elemento difensivo nel panorama dell'architettura militare del Mezzogiorno d'Italia, il donjon (Coppola, 2015, pp. 33-48).

Le fortificazioni degli ex territori longobardi, bizantini e arabi furono adattate alle nuove esigenze di difesa o costruite ex novo secondo tipologie importate dalla Normandia. I castra, cioè spazi chiusi e fortificati, posti sulle cime dei colli, erano atti al controllo, allo sfruttamento del territorio e alla difesa dei nuovi assetti territoriali (Coppola, 2015, pp. 29-77). L'incastellamento normanno in realtà venne a infoltire la già fitta trama castellare longobarda con la costruzione di nuove strutture fortificate, in primo luogo per fissare l'avvenuta conquista del territorio e, successivamente, per rispondere alle immediate esigenze di demarcazione dei confini feudali derivanti dalle nuove acquisizioni territoriali, e per controllare importanti vie di transito (Martin, 1994, pp. 214-216).

Alcuni tra i castelli edificati in epoca normanna sono ancora presenti in elevato e in un discreto stato di conservazione nei seguenti comuni nella provincia irpina: Avellino Ariano Irpino, Calabritto-frazione di Quaglietta, Cervinara, Melito Irpino, Montemiletto, Sant'Angelo dei Lombardi: il dongione e la Torre Febronia, Taurasi, Torella dei Lombardi: la Torre di Girifalco, Nusco e Volturara Irpina (Coppola, Muollo, 2017, pp. 133-136, 143-146, 158-161, 184-186, 228-230, 254-257, 269-271, 310-315, 330-332 pp. 336337, 343-345; Barra, 2013; Coppola, 2012, pp. 90-117). Tutti questi castelli presentano la tipica impostazione quadrangolare o rettangolare del tipico mastio normanno, con o senza muro di spina centrale e con o senza recinto difensivo quadrangolare con quattro torri angolari agli angoli di dimensione ridotta (Coppola, 2015, pp. 29-77; Chatelain, 1973, vedi tavv. IV-V, pp. 6974, 113-124).

In generale, la tipologia del castello normanno in pietra prevedeva la costruzione di una torre principale a pianta rettangolare e a pianta quadrata. Lo sviluppo in altezza prevedeva generalmente 
due o tre piani divisi in ciascun piano con due grandi ambienti attraverso un muro mediano (mur de refend). Sul muro di spina centrale prendevano appoggio i solai, sorretti spesso da volte a botte o a crociera.

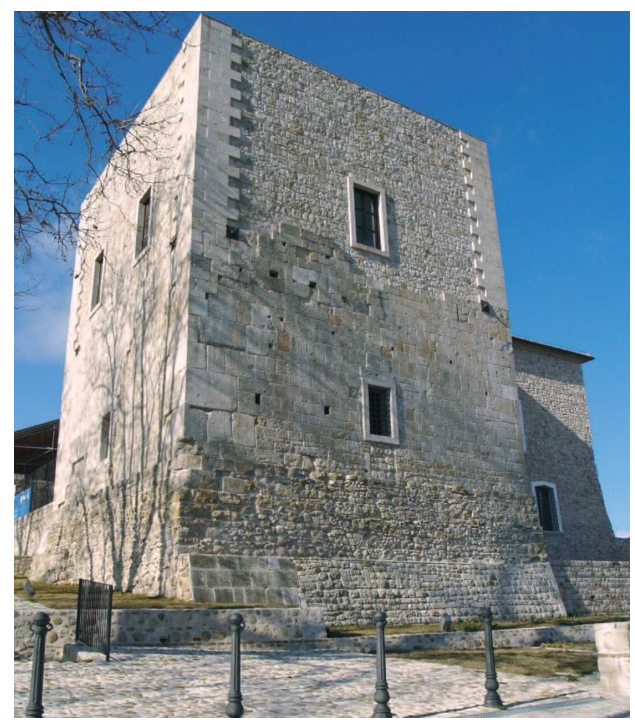

Fig. 2. Sant'Angelo dei Lombardi, donjon, epoca normanna.

Tra le centinaia di masti dell'Italia meridionale ancora osservabili in alzato che conservano intatte tutte le caratteristiche architettoniche dei grandi dongioni d'Oltrealpe ricordiamo tre esempi siciliani, situati in provincia di Catania e costruiti nella prima fase della conquista dell'isola: Adrano, Paternò e Motta Sant'Anastasia (Maurici, 2001, pp. 155, 175176, 178-179).

In definitiva, possiamo affermare che in Italia meridionale dai primi esperimenti di incastellamento longobardo, con valenze esclusivamente strategico-difensive di conquista del territorio, si passerà ad una fase molto più complessa, di tipo militare-feudale, con funzioni sia residenziali che simboliche. In seguito, le semplici tipologie castellari della motta o del donjon andranno assumendo negli anni connotati multiformi, segno di una sperimentazione di soluzioni costruttive che coinvolgerà tutte le strutture del complesso fortificato, dal mastio nelle forme quadrangolari e rettangolari, alla cinta muraria turrita eretta a sua protezione (Coppola, 2015, pp. 67-77).

\section{Torri, castelli e fortificazioni sveve}

Federico II, al rientro dalla Germania, nelle leggi emanate a Capua nel 1220, si occupò della riorganizzazione del sistema difensivo del Regno (Costituzione XIX) (San Germano, 1937-1938, p. 92). Negli anni successivi, rifondato il sistema legislativo (Costituzioni Melfitane, 1231) (Zecchino, 2005, pp. 149-173), l'imperatore avviò l'accertamento giuridico delle comunità e delle persone obbligate, secondo le vigenti consuetudini, alla riparazione dei castelli regi, alla cui manutenzione non era tenuta la Curia regis ( $\mathrm{Pa}-$ sciuta, 2005, p. 454). In base alle indagini in situ effettuate dai funzionari periferici furono censite nel Regnum circa duecentocinquanta strutture non tutte da considerarsi per un uso militare (vi sono elencati castra, domus e palatia). Nel giustizierato, conosciuto con il nome di Principato e Terra Beneventana, in cui rientra l'intera provincia di Avellino, sono individuati 40 castra e 3 domus sul quale insistono i castelli di Bisaccia, di Casalbore e di Solofra, che presentano le tipiche caratteristiche tipologiche federiciane (Coppola, Megna, 2007, pp. 4-19).

La fortificazione di Bisaccia (Coppola, Muollo, 2017, pp. 150-152), come la gran parte dei castelli irpini, ha origine in età longobarda e proprio a questo periodo appartengono alcune strutture murarie dell'impianto del complesso fortificato (seconda metà dell'VIII secolo) messe in luce nei pressi dell'alta torre quadrangolare, il donjon svevo a pianta quadrata con un'altezza di circa $15 \mathrm{~m}$, presenta un evidente connotazione tipologico-stilistica riferibile alla prima metà del XIII secolo. La torre è divisa internamente in tre piani sovrapposti. Per ragioni di sicurezza, la porta d'ingresso originaria è ubicata al primo livello, cui si accedeva presumibilmente mediante un ponte levatoio che poggiava su un pilastro in muratura posto a qualche metro dal muro del dongione. Nonostante la torre di Casalbore (Coppola, Muollo, 2017, pp. 174-176) presenti un'evidente impostazione tipologico-formale di età normanna, il manufatto, a differenza della torre di Cervinara, è da ascrivere alla fase sveva 
per le sostanziali trasformazioni sulle strutture murarie in alzato che non lasciano dubbi per una attribuzione al periodo federiciano attestato da una lapide che reca la data dell'intervento (1216). Tra i diversi esempi di architetture difensive sveve, il castello di Solofra

(Coppola, Muollo, 2017, pp. 322-324), presenta interessanti corpi murari databile all'inizio del XIII secolo con alcuni ambienti principali esposti ad Est che, mediante un piccolo vano gotico preceduto da un corridoio coperto con volte a sesto acuto, permette la comunicazione al donjon di pianta quadrangolare $(7,43 \mathrm{~m} \times 7,54 \mathrm{~m})$ circondato da una cinta della stessa forma con quattro torri ai vertici.

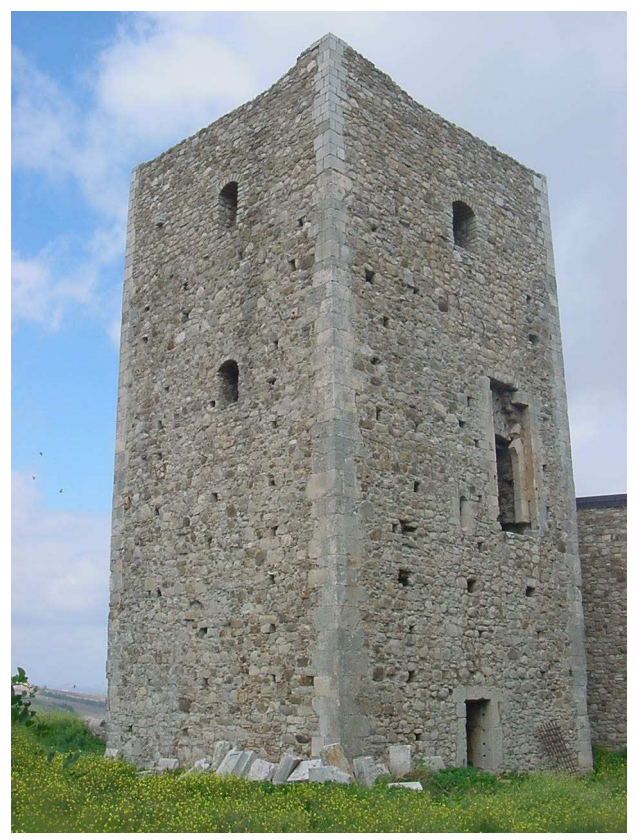

Fig. 3. Bisaccia, torre, epoca sveva.

\section{Torri, castelli e fortificazioni angioine}

Come abbiamo visto, la dominazione sveva apportò notevoli mutamenti all'ordinamento militare strategia che sarà continuata anche dal re Carlo I d'Angiò. I castelli furono allora costruiti con schemi articolati su piante quadrate, rettangolari o poligonali, muniti di torri circolari in corrispondenza degli angoli e di cortine merlate, che si elevavano su alti muri verticali (Santoro,
1978, pp. 21-30). La battaglia di Benevento, combattuta il 26 febbraio 1266 (Iorio, 2016, pp. 9-34), segnò la fine del dominio svevo e l'occupazione di tutto il Regno di Napoli da parte degli Angioini. I castelli furono concessi ai cavalieri che avevano seguito Carlo d'Angiò nella conquista e, sotto l'influenza provenzale, subirono molte modifiche, caratterizzate soprattutto dall'inserimento delle tipiche alte torri rotonde su base troncoconica poste agli angoli della fortificazione. In tal modo l'elemento circolare, tipologia già presente nell'Italia del Nord e nella Francia del re Filippo Augusto (Mesqui, 1991, pp. 162-171), fece la sua apparizione e fu frequentemente usato anche nel Mezzogiorno d'Italia.

Il castello di Monteforte (Santoro, 1985, pp. 8798), costruito dai Longobardi tra fine VI e prima metà del VII secolo per controllare la pianura nolana, fu ampliato dai Normanni e in seguito dagli svevi. Nel 1268 Carlo d'Angiò concesse al conte Guido di Montfort la baronia di Monteforte e le terre di Forino, Atripalda, Cicala e Nola. L'anno seguente iniziarono i lavori del castello con funzioni anche di residenza. Furono incaricati i due noti architetti Pierre d'Angicourt e Jean de Toul che si occuparono, per conto della corona di molti lavori che riguardarono le fortificazioni edificate ex novo o modificate sotto i regnanti angioini. Costituito da una cinta muraria concentrica, in parte ancora visibile, in parte utilizzata come fondazione di abitazioni moderne, del castello restano solo alcuni tratti delle cortine murarie perimetrali di circa $57 \mathrm{~m}$ di lunghezza ed i resti della torre mastio cilindrica con basamento scarpato di età angioina.

Alle costruzioni angioine è da ascrivere, per le sue evidenti connotazioni d'Oltrealpe, la realizzazione del possente torrione circolare di Summonte (Coppola, Muollo, 2017, pp. 327-329), su base leggermente scarpata, collegato al mastio e funzionale per coprire l'ala nord-est. Il castello angioino, si presenta come un alto cilindro con basamento troncoconico costituito da cinque livelli più la copertura e si sviluppa per un'altezza di circa 30 metri. La struttura è molto simile nella forma e nei dettagli costruttivi a quella di molte altre costruite in Campania (Russo, 1999, pp. 183-196; Santoro, 1985, pp. 88-126). 


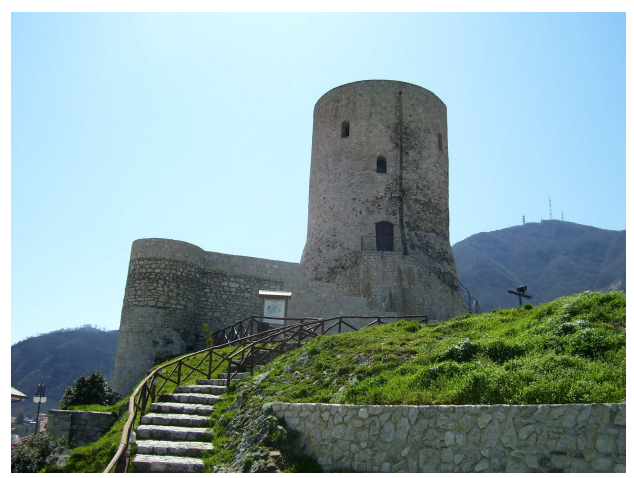

Fig. 4. Summonte, castello, epoca angioina.

Numerose sono in Irpinia le presenze di impianti fortificati angioini: una piccola torre angioina isolata di 4 metri di diametro e di circa 10,50 metri di altezza è ben visibile in altezza a Marzano di Nola (Coppola, Muollo, 2017, pp. 226227); un'altra torre cilindrica svetta all'interno del complesso archeologico del Castello del Monte di Montella (Rotili, Pratillo, 2010) e insieme a un palatium è circondata da un'imponente cinta difensiva; a Rocca San Felice (Rotili, 1991-1992b), situata in un luogo strategico su uno sperone roccioso al limite orientale del ducato beneventano, è presente il donjon cilindrico alto circa 10 metri edificato su precedenti preesistenze, longobarde e normanne. Degno di rilievo è anche il torrione circolare del castello di Avella su base leggermente scarpata, articolato su cinque livelli ${ }^{2}$ e la fortificazione di Ariano Irpino, che dopo la battaglia di Benevento e a seguito dei danni subiti nel 1255 dalla feroce aggressione delle truppe di Manfredi, fu interamente ricostruita in epoca angioina (Coppola, 2012, pp. 90-117, in particolare pp. 109-110). Dello stesso periodo sono poi le cortine murarie del lato Est del castello di Avellino (Barra, 2013, pp. 40-41), attualmente schermato dal muro realizzato dall'intervento dei Caracciolo e una grande torre cilindrica sul lato settentrionale.

L'opera di trasformazione e di restauro degli impianti castellari del precedente periodo svevo, è testimoniata da alcune strutture del castello di Gesualdo (Coppola, Muollo, 2017, pp. 198-200). Analoga situazione di trasformazione e di restauro ad opera di maestranze angioine è ancora ampiamente leggibile nel castello di Taurasi (Cop- pola, Muollo, 2017, pp. 330-332), complesso architettonico fortificato che costituisce un nucleo unico con il borgo abitato.

\section{Torri, castelli e fortificazioni aragonesi}

Con l'insediamento aragonese (Galasso, 1992, pp. 15-301), i castelli subirono molti rimaneggiamenti a causa delle mutate necessità difensive, dovuti dall'impiego generalizzato dell'utilizzo della polvere da sparo che aveva fatto la sua prima apparizione intorno alla fine del XIII secolo fino a diventare nel corso del Quattrocento un imprescindibile strumento di offesa e di difesa (Contamine, 1986, p. 273) dando origine ad una vera e propria architettura militare definita di transizione ${ }^{3}$. Le potenzialità distruttive del cannoneggiamento e l'incredibile efficacia di queste ultime portò all'impiego di bassi e larghi torrioni cilindrici scarpati, all'ispessimento delle mura, alla sostituzione delle fragilissime merlature con robusti merloni, all'utilizzo di opere antemurali con l'impiego di profondi fossati e mura scarpate per permettere la deviazione dei proiettili e quindi una migliore difesa.

Con la decadenza del vicereame spagnolo e la fine della monarchia, la funzione di difesa dei castelli perse l'originaria funzione militare. La presenza di grandi bocche da fuoco per il posizionamento dei cannoni e l'apertura di pareti finestrate ne sono la testimonianza più evidente. Le fortificazioni subirono modifiche sostanziali: molti castelli si trasformarono in vere e proprie residenze signorili, mentre le fortezze situate nei luoghi strategici o poco raggiungibili furono definitivamente abbandonate; alcune furono demolite per il reimpiego di blocchi nelle nuove costruzioni, mentre altre ancora sfidano la forza edace del tempo, l'incuria degli uomini e le frequenti scosse dei terremoti (Maglio, 2001).

In provincia di Avellino, gli interventi vicereali mutarono la configurazione di molti castelli preesistenti, quasi sempre d'epoca angioina, determinando spesso un ibrido fortificato che presenterà in Italia meridionale un impianto tipologico del tutto particolare con evidenti difficoltà di datazione. A questo gruppo appartengono i castelli (Coppola, Muollo, 2017): Ariano Irpino, 
Gesualdo, Grottaminarda, Grottolella, Manocalzati, Melito Irpino, Montefalcione, Montemiletto, Monteverde, Sant'Andrea di Conza, Taurasi, Torella dei Lombardi, Tufo e Zungoli.

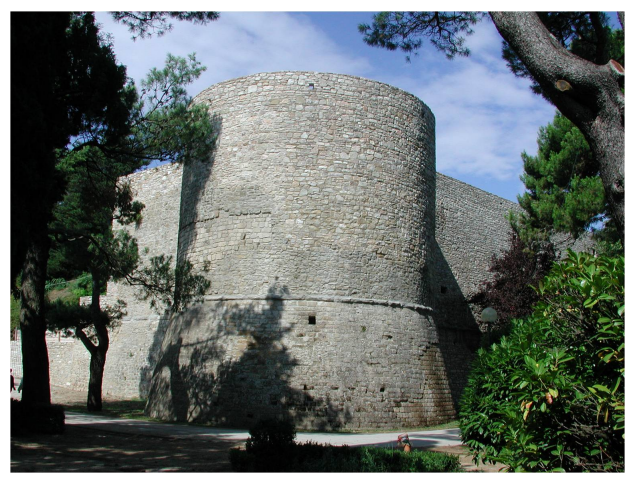

Fig. 5. Ariano Irpino, castello, epoca aragonese.

Nella parte occidentale dell'Irpinia vi sono due castelli che conservano ancora parti d'epoca angioina alle quali si sovrappongono, a volte incamiciandole, elementi di chiara matrice aragonese. Si tratta del castello di San Barbato a Manocalzati e del castello di Montefredane che gli fa pendant sul crinale opposto.

La fase aragonese risulta tuttora dominante nell'aspetto esterno del complesso fortificato di Ariano Irpino: dopo il sisma del 1456, furono elevate, in posizione sostanzialmente simmetrica alle estremità del recinto murario, le quattro torri circolari scarpate che inglobarono, come si è avuto modo di dire, le precedenti torri angioine (Coppola, 2012, pp. 109-110). Le torri, di altezza pari a quella delle cortine murarie, sono realizzate secondo canoni costruttivi tipici dell'edilizia di fine Quattrocento quali appaiono, per esempio, nelle strutture aragonesi del Castelnuovo di Napoli (Coppola, 2012, pp. 109110; Santoro, 1985, pp. 140-168).

Con forma trapezoidale, sempre di impianto aragonese, è anche il castello di Grottaminarda (Coppola, Muollo, 2017, pp. 201-203). Un ulte- riore esempio della stessa tipologia è data dal castello di Lacedonia (Coppola, Muollo, 2017, pp. 210-212), costruito nel primo decennio del 1500 da Ferdinando Pappacoda, che aveva ottenuto la terra di Lacedonia da Federico d'Aragona nel 1496. Il castello a pianta quadrata, presenta due torri cilindriche su base scarpata, con cortine interposte e una terza torre sul lato Est di forma lanceolata, molto simile ad un'altra torre riscontrabile nel vicino castello di Rocchetta Sant'Antonio (Rescio, 1999, pp. 211-226), costruita negli stessi anni, dove non è difficile riconoscere l'inconfondibile stile dell'architettura militare rinascimentale di Francesco di Giorgio Martini (Dechert, 1990, pp. 161-180). Ma da qui in poi l'architettura militare in Italia meridionale segue un' altra storia e iniziano i tempi moderni ${ }^{4}$.

\section{Note}

1 "Le fortezze erano simboli letterali e figurativi di difesa contro la potenziale invasione straniera, derivati da una strategia militare e un' architettura che seguivano una moda duplicata in tutta Europa": Hogg, 1982, p. 8.

Sugli insediamenti difensivi longobardi in Italia meridionale: Peduto, Natella, 2007, in particolare le pp. 362-369; Rotili, 2003.

${ }^{2}$ La torre circolare, già presente in Francia e nell'Italia del Nord, fu introdotta nel meridione d'Italia in età angioina ed ebbe rapida diffusione, si veda: Mesqui, 1991, pp. 162-171; Santoro, 1985, pp. 45-52; Licinio, 1994, pp. 228-246.

${ }^{3}$ Per la Campania si veda: Russo, 1999, pp. 183196. Per gli esempi italiani ed europei si consulti: Cassi Ramelli, 1996, pp. 309-384.

${ }^{4}$ Sulle fortificazioni in epoca moderna si vedano gli importanti lavori di: Viglino Davico, Lusso, 2013, pp. 60-71; e soprattutto il lavoro di A. Fara (1997), per quanto riguarda la parte riservata al passaggio dell'ingegnere militare medievale a quello dei tempi moderni.

\section{Bibliography}

Barra, F. (2013). Il castello di Avellino, Avellino.

Brogiolo, G.P.; Marazzi, F.; Giostra, C., eds. (2017). I Longobardi. Un popolo che cambia la storia, Skira Ed., Milano. Cassi Ramelli, A. (1996). Dalle caverne ai rifugi blindati. Trenta secoli di architettura militare, Bari. 
Chatelain, A. (1973). Donjons romans des pays d'ouest, Parigi.

Cinquantaquattro, T.; Camardo, D.; Basile, F. (2003). "Il castello di Avella (AV): le indagini archeologiche sulla rocca", in Atti del III Convegno Nazionale di Archeologia medievale, Firenze.

Colantuono, A. (1993). "Monticchio dei Lombardi: il castello, il feudo, il borgo medievale", in Civiltà Altirpina, s. IV.

Contamine, P. (1986). La guerra nel Medioevo, Bologna.

Coppola, G.; Megna, C. (2007). "Tre castelli normanno-svevi in Irpinia: Bisaccia, Cervinara, Solofra”, L'Irpinia illustrata, $2 / 24$.

Coppola, G.; Muollo, G. (2017). Castelli medievali in Irpinia. Memoria e conoscenza, Napoli.

Coppola, G. (2005). L'architettura dell'Italia meridionale in età normanna, secoli XI-XII, Napoli.

Coppola, G. (2012). Il castello medievale di Ariano, Zecchino, F. dr., Ariano Irpino.

Coppola, G. (2015). Battaglie normanne di terra e di mare. Italia meridionale secoli XI-XII, Napoli.

Cucciniello, A. (2012). "Capolavori della Terra di Mezzo. Opere d'arte dal Medioevo al Barocco", in Catalogo della mostra, Avellino, ex carcere Borbonico, 28 aprile-30 novembre 2012, Napoli.

Cuozzo, E. (1985). Catalogus Baronum: Commentario, Fonti per la Storia d'Italia, 101**, Roma.

Dechert, M.S.S. (1990). "The military architecture of F. di G. in Southern Italy", Journal of the Society of architectural historians, 49, 2.

Erchemperto. (1999). Historia Langobardorum Beneventanorum, XVIII, Waitz, G. dr., Matarazzo, R. trad., Napoli.

Fara, A. (1997). Bernardo Buontalenti, Milano.

Galasso, G. (1992). Il Regno di Napoli. Il Mezzogiorno angioino e aragonese (1266-1494), Storia d'Italia, XV, tom. I.

Gambino, N. (1977). "Sant'Angelo al Pesco, profilo storico: prima parte”, in Civiltà Altirpina, s. II, II.

Gandolfo, F.; Muollo, G. (2013). Arte medievale in Irpinia, Roma.

Gasparri, S. (1995). "La frontiera in Italia (sec. V-VIII). Osservazioni su un tema controverso", in Brogiolo, G.P. ed., Città, castelli, campagne nei territori di frontiera (secoli VI-VII), Mantova.

Hogg, I. (1982). Storia delle fortificazioni, Novara.

Iorio, G. (2016). "La battaglia di Benevento (26 Febbraio 1266) nei cronisti coevi”, Schola Salernitana. Annali, 21.

Jamison, E., ed. (1972). Catalogus Baronum, Fonti per la Storia d'Italia, 101, Roma.

Johannowsky, W. (2000). "Risultati della ricerca archeologica a Compsa e nell'ager Compsanus", in VV.AA., Memorie Conzane vol. II, parte I. Atti dell'incontro-dibattito del 3 maggio 1981 su Il terremoto del 23 novembre e la rinascita di Conza, Compsa, pp. 31-38.

Licinio, R. (1994). Castelli medievali. Puglia e Basilicata: dai Normanni a Federico II e Carlo d'Angiò, Bari.

Maglio, L. (2001). Architetture fortificate vicereali spagnole nel regno di Napoli nei secoli XVI e XVII, Napoli.

Martin, J.M. (1992). “Les problèmes de la frontière en Italie méridionale (Vie-XIIe): l'approche historique”, in Frontière et peuplement dans le monde méditerranéen au Moyen Age, Poisson, J.M. ed., Rome-Madrid.

Martin, J.M. (1994). “L'impronta normanna sul territorio, in I Normanni Popolo d'Europa, 1030-1200”, in D’Onofrio, M. ed., Catalogo della mostra, Roma-Palazzo Venezia, 28 gennaio-30 aprile 1994, Venezia, pp. 214-216.

Martin, J.M. (2005). Guerre, accords et frontières en Italie méridionale pendant le haut Moyen Âge: Pacta de Liburia, Divisio principatus Beneventani et autres actes, Roma.

Maurici, F. (2001). Castelli medievali di Sicilia. Guida agli itinerari castellani dell'isola, Palermo.

Mesqui, J. (1991). Châteaux et enceintes de la France médiévale, Paris, tom. I.

Pasciuta, B. (2005). "Curia regis”, in Federico II. Enciclopedia Fridericiana, Roma, vol. I.

Peduto, P.; Natella, P. (2007). "Insediamenti Longobardi nel ducato di Benevento (secoli VI-VIII)", in Gasparri, S.; Cammarosano, P. eds., Langobardia, Udine.

Putaturo Donati Viscido di Nocera, M. (2008). Profili di storia feudale della terra di Quaglietta nella valle del Sele, Nusco.

Rescio, P. (1999). "Il castello e il centro storico di Rocchetta Sant'Antonio", in Archeologia e Storia dei castelli di Puglia e Basilicata, Soveria Mannelli. 
Rotili, M. (1991-1992a). "Ricerche archeologiche nel castello di Rocca San Felice", Rendiconti dell'Accademia di Archeologia, Lettere e Belle Arti in Napoli, 63, pp. 231-384.

Rotili, M. (1991-1992b). "Rocca San Felice: ricerche archeologiche 1990-1992”, Rendiconti dell'Accademia di Archeologia, Lettere e Belle Arti in Napoli, 63, pp. 231-384.

Rotili, M. (2002). Sant'Angelo dei Lombardi: ricerche nel castello (1987-96), Archeologia castellana nell'Italia meridionale, Napoli.

Rotili, M. (2003). "Benevento e il suo territorio: persistenze e trasformazioni", in I Longobardi dei ducati di Spoleto e Benevento, Atti del XVI Congresso internazionale di studi sull'Alto Medioevo, Spoleto-Benevento 20-27 ottobre 2002, Todi, pp. 827-879.

Rotili, M.; Pratillo, P. (2010). "Il castello del Monte di Montella. Ricerche archeologiche 1980-1992, 2005-2008”, in Patitucci Uggeri, S. ed., Archeologia castellana nell'Italia meridionale. Bilanci e aggiornamenti, Palermo, pp. 167-180.

Russo, F. (1999). Trenta secoli di fortificazioni in Campania, Napoli.

San Germano, R. di. (1937-1938). Chronica, Garufi, C.A. ed., N. Zanichelli Ed., Bologna.

Santoro, L. (1978). "I castelli angioini della Campania”, Castellum, 19.

Santoro, L. (1985). Castelli angioini e aragonesi nel regno di Napoli, Milano.

Toubert, P. (1997). Dalla terra ai castelli. Paesaggio, agricoltura e poteri nell'Italia medievale, Torino.

Viglino Davico, M.; Lusso, E. (2013). "L’ingegneria delle difese militari”, in Enciclopedia Italiana-Treccani, Il contributo italiano alla storia del pensiero, Tecnica, Roma.

VV.AA. (2003). Longobardi dei ducati di Spoleto e Benevento: atti del XVI Congresso Internazionale di Studi sull'Alto Medioevo; Spoleto, 20-23 ottobre 2002, Benevento, 24-27 ottobre 2002, Spoleto, 2 vols.

Zecchino, O. (2005). “Liber Constitutionum”, in Federico II Enciclopedia Fridericiana, Roma, vol. II. 
